\title{
Ultrasound visualization of anatomical structures through a sterile transparent dressing
}

\author{
Michael Hogan, MBBCh • Timothy Shakespeare, MBBS • \\ Ban Tsui, MD
}

Received: 28 September 2012/ Accepted: 4 October 2012/Published online: 22 December 2012

(c) Canadian Anesthesiologists' Society 2012

\section{To the Editor,}

We read with great interest the article entitled "Use of a chlorhexidine-impregnated patch does not decrease the incidence of bacterial colonization of femoral nerve catheters: a randomized trial" ${ }^{1}$ published in the Journal, as we have recently started using a similar anti-bacterial gel dressing with the aim to minimize risk of infection during continuous peripheral nerve catheterization. Although it was disappointing to learn that there is no evidence of reduced femoral nerve catheter infection with the use of the BIOPATCH $^{\circledR}$ (Ethicon Inc., Somerville, NJ, USA), we would like to alert readers to our observation of a potential unrelated benefit of chlorhexidine-impregnated gel dressings, specifically, allowing ultrasound visualization of underlying anatomical structures.

Ultrasound imaging is gaining popularity as a method of continuous peripheral nerve localization and central venous line placement; however, it is common experience that visualization of subcutaneous anatomical structures is almost impossible through an applied dressing. This is because most standard dressings consist of gauze-like materials that allow air tracking between the probe and the dressing, which interferes with ultrasound visualization.
We noticed recently that we were able to obtain effective ultrasonographic visualization of anatomical structures using a sterile transparent dressing with a chlorhexidineimpregnated gel component (Tegaderm $^{\mathrm{TM}}$ CHG, 3M Health Care, St. Paul, MN, USA). To illustrate, we compared the ability to visualize the median nerve of the author's mid-forearm with and without the dressing (Figure). To our amazement, the image obtained through the dressing was of noticeably similar quality when the overall gain was increased. Upon closer examination, the dressing consists of Tegaderm and a thin gel layer, providing a smooth, air-free interface. Conversely, other commonly used gauze-based dressings create multiple small air pockets under the adhesive dressing.

This observation illustrates the assertion that ultrasound waves emitted from probes are prone to attenuation by poor contact, air, and subtle changes in interface materials. This letter serves to remind clinicians that certain dressings (e.g., Tegaderm CHG; Comfeel Plus, Coloplast ${ }^{\circledR} \mathrm{A} / \mathrm{S}$, Humlebæk, Denmark) allow ultrasound imaging, and in order to obtain the best quality image, close attention should be paid to any media placed between the probe and patient. $^{2}$ 
Figure Comparison of ultrasound imaging of the median nerve in the mid-forearm through a chlorhexidine gel dressing (left) and without a dressing (right). The gel dressing as it appears on ultrasound is also indicated (bottom left panel)
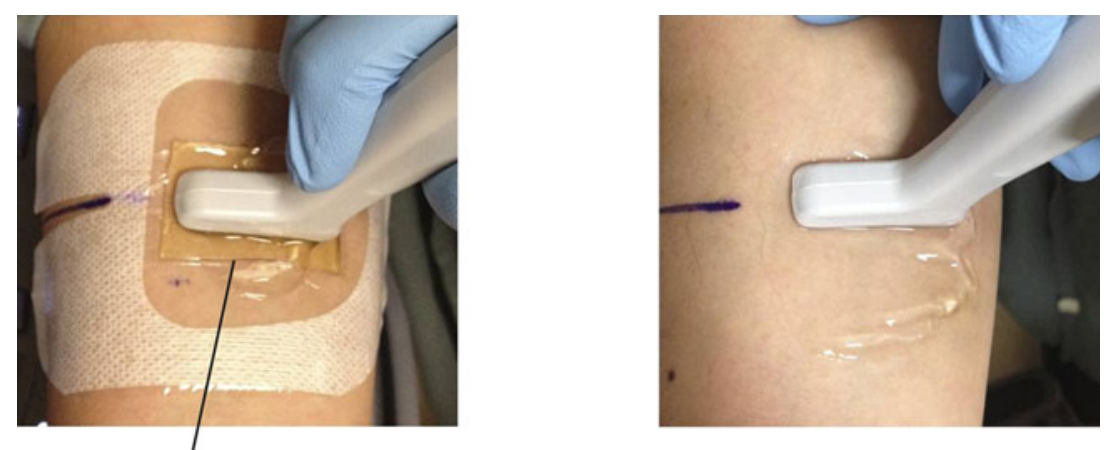

Chlorhexidine gel dressing
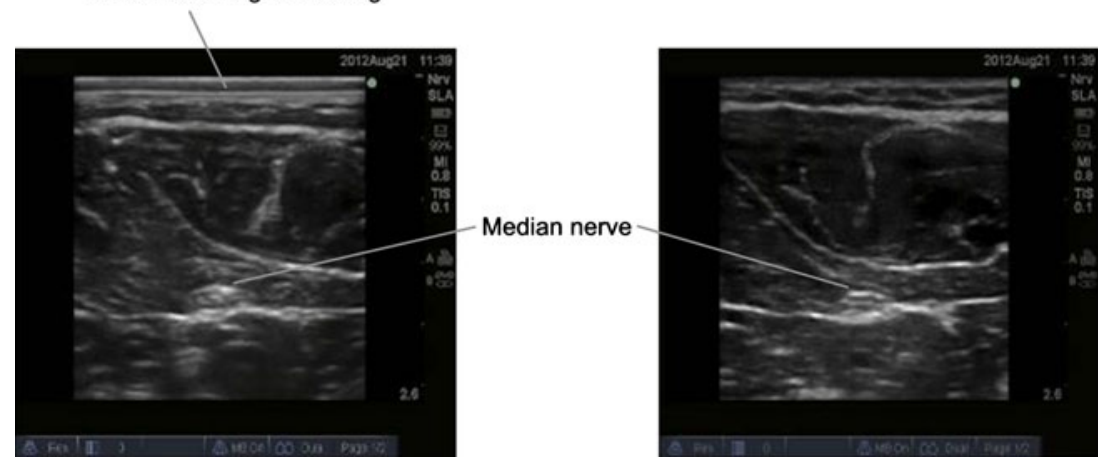

Funding The corresponding author (B.T.) is supported in part by a Clinical Scholar Award from the Alberta Heritage Foundation for Medical Research (AHFMR) and a CAS/Abbott Laboratories Career Scientist Award from the Canadian Anesthesiologists' Society.

Conflicts of interest/other associations The corresponding author (B.T.) has a patent licensing agreement with Pajunk; however, there is no conflict of interest concerning the equipment used in this study. The other authors declare no conflict of interest.

\section{References}

1. Schroeder KM, Jacobs RA, Guite C, Gassner K, Anderson B, Donnelly $M J$. Use of a chlorhexidine-impregnated patch does not decrease the incidence of bacterial colonization of femoral nerve catheters: a randomized trial. Can J Anesth 2012; 59: 950-7.

2. Tsui BC, Twomey $C$, Finucane BT. Visualization of the brachial plexus in the supraclavicular region using a curved ultrasound probe with a sterile transparent dressing. Reg Anesth Pain Med 2006; 31: 182-4. 